tionner en parallèle si la section du cuivre devaient insuffisante ou si un transformateur venait à faire défaut.

Les branchements des abonnés et de l'éclairage public sont exécutés comme pour le réseau périphérique. Toutefois les lampes publiques fixées sur poteaux de bois, sont reliées à la ligne par du câble sous-plomb de 600 méghoms, logé dans une moulure de bois goudronné, vissée elle-même au poteau.

Le montage des postes de transformateurs du réseau suburbain, ainsi que les constructions des lignes aériennes du réseau périphérique et de la banlieue, ont été exécutés par la Société Grenobloise d'Electricité.

\section{RÉSUMÉ GÉNÉRAL}

Le secteur de Grenoble comprend donc 2 réseaux différents :

Le réseau urbain comprend le réseau du centre et celui de la périphérie. Celui du centre comprend 35 postes de transformateurs montés dans des tourelles métalliques, alimentant des lignes secondaires souterraines.

Le réseau périphérique comprend 18 postes montés dans des kiosques en ciment armé, alimentant des câbles secondaires souterrains, mais surtout des lignes aériennes.

Le réseau suburbain comprend i 2 postes montés dans des kiosques-pylônes en tôle d'acier, alimentant de même quelques lignes souterraines, mais principalement des lignes aériennes.

En debors de ceci, il existe encore 12 postes de transformateurs installés, soit dans des bâtiments communaux (Ecoles), soit chez de gros clients d'éclairage.

Le réseau municipal comporte donc actuellement 77 postes de transformateurs; d'après la description donnée précédemment, les allumeurs ont donc 65 rotatifs à manœuvrer, pour procéder à l'éclairage de tout le territoire de Grenoble.

Les abonnés d'une force motrice supérieure à $3 \mathrm{HP}$ ont chez eux des transformateurs qui sont leur propriété; ceux-ci sont actuellement au nombre de 35 .

La puissance totale des $77+35=112$ transformateurs reliés au secteur de Grenoble est environ de $5400 \mathrm{kw}$., représentant une consommation à vide de $70 \mathrm{kw}$. environ.

$\mathrm{Au} 30$ avril 1906, il y avait environ l'équivalent de 30000 lampes de to bougies installées chez les particuliers; à celles-ci, il convient d'ajouter environ 220 lampes à arc et I 600 lampes d'escaliers.

Les abonnés de force motrice, au nombre de 150 environ, absorbent une puissance d'environ i $200 \mathrm{HP}$ aux bornes des moteurs.

La puissance de ces moteurs varie à l'infini de I/ Io à ıoo HP. Sauf un seul, de $85 \mathrm{HP}$, qui est alimenté directement à $b$ ooo volts, ils fonctionnent tous à I 20 volts.

Les bâtiments communaux ont nécessité l'installation de 3500 lampes à incandescence et de 20 lampes à arc.

Le réseau d'éclairage public comporte environ r 500 lampes à incandescence de 16 bougies, et 225 lampes à arc. Ces dernières présentent un certain intérêt au point de vue des charbons qui sont minéralisés; ils sont dus à M. Blondel. La puissance lumineuse hémi-sphérique de ces lampes, dont la consommation moyenne est de 400 watts, y compris le rendement du transformateur, est de 2000 bougies environ. Elles fonctionnent à un ampèrage de 14 à 15 ampères, sous 29 à 30 volts; chaque lampe est branchée en dérivation sur le câble éclairage public par l'intermédiaire d'un petit transformatcur, dont le rapport de transformation est de $120 / 30$ volts environ en charge, et I $20 / 44$ volts à vide. Cette chute de tension, qui paraît énorme, est indispensable au bon fonctionnement de l'arc; on évite de cette façon des débits anormaux lorsque les charbons sont au calage.

Enfin, avant de vous rendre la liberté, je crois intéressant de vous indiquer encore les longueurs des différentes sortes de lignes constituant notre secteur municipal :

Câbles à 5000 volts................... 3o kil. Câbles à 120 volts Eclairage particulier 3 fils..... 30 - - - public - 35 Lignes aériennes I 20 volts Eclairage particulier 3 fils. $20-$ Lignes aériennes - - - public - 24 Câbles à 120 volts banlieue, 2,3 et 4 fils....... 7 -

J'espère, Messieurs, que ces explications, qui ont peutêtre été un peu longues, vous auront donné une idée du Service Electrique de la Ville, que nous nous efforcerons sans cesse de perfectionner, car notre plus ardent désir est de faciliter la tâche de ces vaillants industriels qui font la réclame et la gloire de cette Ville de Grenoble, qui est à la tête des cités de lumière, marchant si courageusement au progrès, et concourant ainsi à la prospérité et à l'honneur de la nation française.

\section{ÉTUDE SUR LES}

\section{BHRRHGES EN MAÇONNERIE \\ et Murs de Réservoirs}

(suite)

\section{Calcul des barrages a profil triangulaire}

Parmi tous les profils que l'on peut imaginer, c'est celui dont le parement amont est vertical qui, à volume égal, donne en charge les meilleures conditions de stabilité; ou, inversement, et à conditions égales de stabilité, c'est celui

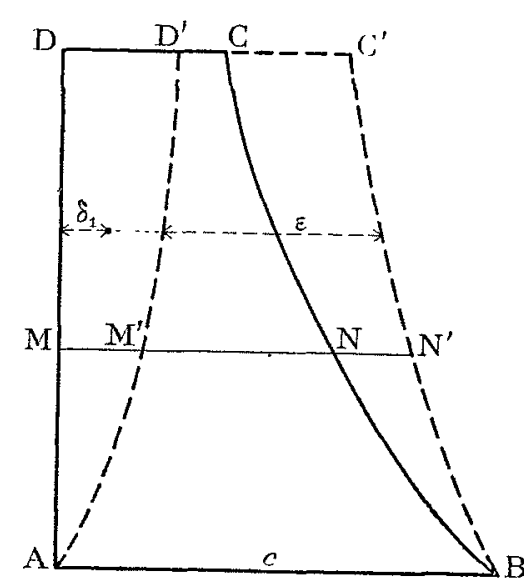

Fig. 69. qui est le plus économique. Ceci résulte immédiatement des considérations suivantes :

Soit $A B C D$ et $A B C$ 'D' deux profils ayant même hauteur, et, pour chaque section horizontale telle que $\mathrm{MN}$, même largeur, ce qui entraîne par suite même volume. Désignons par $N$ le poids des maçonneries de l'un et l'autre profil, par $\delta$ et $\delta$ ' les distances à la verticale AD des centres de gravité des deux profils, par $p$ le poids de l'eau correspondant au triangle ADD' dont le centre de gravité est à une distance $\delta_{1}$ de la verticale $\mathrm{AD}$, et par $e$ la largeur de la base $\mathrm{AB}$. Les pressions $n_{v}$ pour le profil $\mathrm{ABCD}$, et $n_{2}$ pour le profil ABC'I', ont pour valeur:

$$
\begin{aligned}
& n_{v}^{\prime}=\frac{N}{e}+6 \frac{M_{2}}{e^{2}}-\frac{y^{3}}{e^{2}}=\frac{2 N\left(2 e-3 \delta^{2}\right)}{e^{2}}-\frac{y^{3}}{e^{2}} \\
& n_{t}^{\prime}=2 \frac{N+p}{e^{2}}\left(2 e-3 \frac{N \delta^{\prime}+p \delta_{1}}{N+p}\right)-\frac{y^{3}}{e^{2}}
\end{aligned}
$$


on en tire :

$$
n_{v}^{\prime}-n_{1}^{\prime}=2\left[\frac{3 N\left(\delta^{\prime}-\delta^{\prime}\right)+p\left(3 \delta_{1}-2 e\right)}{e^{2}}\right]
$$

Or, si l'on désigne par $\varepsilon$ la largeur du joint de l'un et l'autre profil sur la section horizontale passant par les centres de gravité des surfaces ADD' et BCC', on a :

ce qui donne :

$$
N\left(\delta^{\prime}-\delta\right)=K p \varepsilon
$$

$$
n_{v}^{\prime}-n_{1}^{\prime}=6 \frac{p}{e^{2}}\left(\delta_{1}+K_{\varepsilon}-\frac{2}{3} e\right)
$$

mais l'on a presque toujours $\varepsilon>\frac{e}{3}$, en outre $K>2$, de sorte que l'on peut dire que $n_{v}^{\prime}$ est, en pratique, toujours plus grand que $\imath^{\prime}$, et qu'il l'est d'autant plus que le profil ABC'D' s'écarte plus du profil $A B C D$, puisque o et $p$ croissent lorsque AD' s'écarte de $\mathrm{AD}$.

On trouverait aussi que l'on a en $\mathrm{B}$, sur le parement aval:

$$
n^{\prime}{ }_{1}-n_{v}{ }_{v}=6 \frac{p}{e^{2}}\left(\delta_{1}+K \varepsilon-\frac{e}{3}\right)
$$

Comme précédemment, et à plus forte raison, on voit que $n^{\prime}{ }_{i}$ est en pratique toujours plus grand que $n{ }^{\prime \prime}$, et d'autant plus que le parement amont s'écarte plus de la verticale.

En réalité, ce qu'il importe de rendre minimum, ce n'est pas tant $n$ ", que la compression maxima ' $n{ }_{2}$ ou $A$. Or, en inclinant le parement amont à la base, on redresse celui d'aval, el par suite l'on diminue bien $\operatorname{tg}^{2} \beta$, mais en même temps on augmente $n^{\prime \prime}$, de sorte que, dans la généralité des cas, $A$ reste minimum lorsque le parement amont est très sensiblement vertical. Nous l'avons d'ailleurs vérifié pour le profil triangulaire $\left({ }^{\star *}\right)$.

Comme l'on cherche d'abord à obtenir la plus grande pression possible à l'amont en charge, afin d'éviter le danger des sous-pressions, et ensuite la plus faible compression à l'aval lorsque la hauteur est un peu grande, afin de s'éloigner de la résistance limite pratiquement acceptable pour les maçonneries, on voit donc que le profil à parement amont vertical est celui qui est le plus avantageux.

Pour qu'un barrage puisse être certainement soustrait au dangel des sous-pressions, il faut que la pression $n$ ' des maçonneries à l'amont en charge soit au moins égale à celle de l'eau. Or, pour que cette condition puisse être réalisée de la façon la plus économique, il faut que le volume des maçonneries employées soit le plus petit possible, ce qui conduit à faire passer la courbe des pressions en charge aussi près qu'on le pourra du parement amont, ce qui oblige aussi à faire également passer ia courbe des pressions à vide aussi près que possible de l'amont, et, comme elle ne doit pas pénétrer dans le tiers amont, si l'on veut éviter tout effort de traction à l'aval à vide, il faut que cette courbe passe juste par le tiers amont. Cette combinaison présente encore l'avantage de rendre minima

(') En effet, comme l'on a $M N=\mathrm{M}^{\prime} \mathrm{N}^{\prime}$, on a aussı $\mathrm{MM}$ ' $=\mathrm{NN}$ ', ainsı que surf. $\mathrm{ADD}^{\prime}=$ surf. BCG'. Or, le produrt $N\left(\delta^{\prime}-\delta\right)$ représente le traval qu'll faudralt dépenser pour transporter le poids $N$ de l'un ou l'autre profil sur la distance $\left(\delta^{\prime}-\delta\right)$, ou bien encore pour amener le protil $A B C D$ dans la position $A B C D^{\prime}$; mats ce travail est aussi égal à cclur qu'il faudralt effectuer pour transporter la surface AD'D dans la position $\mathrm{BC} \mathrm{C}^{\prime}$, c'est-à-dıre égal à $K p \varepsilon$, z étant la distance des centres de gravité des deux surfaces. $\varepsilon$ est aussi la largeur du joint de l'un et lautre profil sur la section horizontale passant par ces centres de gravités, ce qu'il est facile de vérifier sı l'on remarque que, pour chaque section telle que MN', la distance des centres de gravité de MM' $^{\prime}$ et de NN' est égale à la largeur du joint, MN ou M'N', de l'un ou l'autre probil.

(“) Volr La Houille Blanche, $\mathrm{n}^{\circ}$ de juin 1905, et aussi les Annales des Ponts el Chaussées, 1889 M. Banbet, Résistance des barrages en magonneries. la pression normale $n$ " à l'aval. Or, la condition précédente est précisément réalisée par le profil triangulaire ì parement amont vertical ; ce profil parait donc de prime abord comme très avantageux. Mais, en pratique, il est absolument impossible de construire un barrage parfaitement triangulaire, car il faut toujours donner aux maçonneries une certaine épaisseur au sommet, soit à causc des exigences de la construction, soil afin de permettre au couronnement de résister au choc des vagues ou à la poussée des glaces, soit enfin pour servir de voie de communication entre les deux côtés de la vallée. Le profil pratique doit done s'écarter quelque peu du profil triangulaire, et cela d'autant plus que le barrage est moins haut, mais nous allons voir plus loin que, bien loin d'être une gêne, cette épaisseur obligatoire en couronne peut, au contraire, apporter un surcroit de stabilité ou une économie dans le volume des maçonneries.

Le profil trapézoidal n'est pas le plus avantageux, car sa courbe des pressions à vide passe à l'intérieur du tiers médian de la base.

En outre du profil triangulaire théorique, il est possible de trouver d'autres profils pour lesquels la courbe des pressions à vide passe très près du tiers amont de la base. On peut ainsi réaliser un profil se rapprochant de celut d'égale résistance de M. Delocre, mais ce profil n'apparait pas comme le plus avantageux. En effet, son parement aval est une courbe tournant sa convexité vers l'amont, et pénétrant à l'intérieur du profil triangulaire pratique équivalent; par suite, si le parement amont est vertical, ce profil fait passer la courbe des pressions à vide dans le tiers amont, ce qui occasionne des efforts de traction à l'aval. Pour éviter ces efforts, il faut donner du fruit au parement amont, mais nous venons de voir que cette disposition n'est pas avantageuse; le profil Delocre paralt done moins pratique que le profil triangulaire. De plus, la courbe du parement aval fait à la base avec la verticale un angle plus grand que l'angle du triangle correspondant, de sorte que la valeur de tgô, et par suite celle de la compression maxima $A$, se trouve plus élevée dans le cas du profil Delocre que dans celui du triangle. Cet inconvénient est surtout ennuyeux pour les ouvrages de très grande hauteur pour lesquels est on amené, afin de diminuer la valeur de $\operatorname{tg}^{2} \beta$, à redresser le parement aval vers la base, ainsi quon le verra plus loin, et à donner au profil une forme se rapprochant de ceux de MM. Guillemain et Hétier.

Le profil général triangulaire parait donc comme le plus avantageux au point de vue économique. Il a encore un autre mérite, c'est qu'il permet d'établir des relations simples entre ses dimensions et les efforts auxquels sont soumises les maçonneries; aussi allons-nous l'étudier d'une manière toute spéciale. Pour cela, nous considèrerons un profil triangulaire fondamental BAS, surmonté d'un autre triangle SCD inversement placé, et formant couronnement.

Désignons par $e_{0}$ la largeur du couronnement SC, par $y_{0}$ la hauteur $\mathrm{CD}$ du triangle de couronnement,par $c$ la largeur de la base $\mathrm{AB}$, et par $m$ la distance AP du pied de la verticale SP du sommet $S$ du triangle fondamental. La distance $d d u$ centre de gravité du mur à vide au point A est :

$$
d=\frac{e}{3} \cdot \frac{1-\mu^{2}+n^{2}(3 \mu+2 n)}{1-\mu+n^{2}}
$$

en posant

$$
\frac{m}{e}=\mu \quad \text { et } \quad \frac{e_{0}}{e}=n
$$


Pour une valeur déterminée de $\mu$, le minimum de $d$ a lieu lorsque la dérivée de cette expression, prise par rapport à $n$, est nulle.

Dans le cas particulier où $\mu=0$, c'est-à-dire lorsque le parement amont est vertical, ce minimum a lieu lorsque n satisfait à la relation :

$$
n^{3}+3 n-1=0
$$

qui a pour racine $n=0,322$, soit un tiers en nombre rond, ce qui donne pour le minimum de $d$ la valeur :

$$
d_{0}=\frac{29}{30} \cdot \frac{e}{3}
$$

Ainsi, par le fait de l'adjonction du triangle SCD, le centre de gravité du triangle fondamental peut se trouver reporté vers l'amont de $1 / 90$ de $e$, et la force qui représente le poids du système ne passe plus par le tier's midian de l'ouvrage. Ce qui, avec la loi du trapèze, correspoud sur le parement aval, à un effort maximum de traction sensiblement égal à :

$$
\left(\frac{1}{15} \cdot \frac{N}{e}\right)\left(1+\operatorname{tg}^{2} \beta\right)
$$

Pour éviter toute trac-

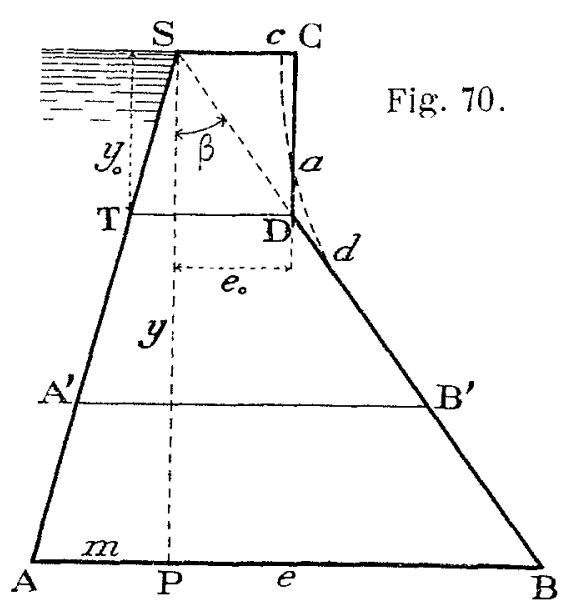
tion à l'aval, il faut done donner au parement amont une légère inclinaison.

Pour une section horizontale qnelconque A'B', si l'on veut que la distance $d$ soit égale au tiers de la largeur A'B', il faut que $\mu$ et $\eta$ satisfassent à la relation :

$$
\mu^{2}-\left(1+3 n^{2}\right) \mu+n^{2}(1-2 n)=0
$$

Mais, si le rapport $\mu$ reste constant sur toute la hauteur du mur comprise entre le massif de couronnement et la base, il n'en est pas de même pour $n$ qui décroit au fur et à mesure que la section variable A'B' se rapproche de la base AB. Il faut donc donner à $\mu$ la plus grande valeur qu'il soit susceptible de prendre, c'est-à-dire celle qui résulte de la résolution de léquation $\frac{\mathrm{d} \mu}{\mathrm{d} n}=0$, qui donne $n=\frac{1}{3}-\mu$

et

$$
\mu^{3}-2 \mu^{2}+\frac{4}{3} \mu-\frac{1}{27}=0
$$

dont la racine est $\mu=0,029\left({ }^{*}\right)$, soit 0,03 en nombre rond.

Pour toute valeur de $\mu \geqslant 0,03$ on aura toujours $d \geqslant e / 3$ et l'inclinaison du parement sera ainsi déterminée tant que la hauteur de l'ouvrage ne sera pas assez grande pour nécessiter un renforcement de la largeur à la base.

Il est facile de trouver la hauteur $h$ jusqu'à laquelle le parement amont peut rester vertical sans qu'il en résulte d'effort de traction à l'aval à vide. En effet, tant que la verticale du centre de gravité du triangle de couronnement SCD, dont la distance au parement amont est $2 / 3 e_{0}$,

(*) Cette racine s'obtient immédlatement en faisant disparaître le terme $\mu^{2}$. En effet, si l'on pose:

léquation donnée se réduit à :

$$
\mu=\frac{2}{3}+\xi
$$

$$
\xi^{3}+\frac{7}{27}=0 \quad \text { d'où }: \quad \mu=\frac{1}{3}(2-\sqrt[3]{7})=0,029
$$

tombe à l'intérieur du tiers médian de la base, la courbe des pression: à vide passe elle-même à l'intérieur. La hauteur $h$ est donc celle pour laquelle les centres de gravité des triangles SCD et SAB se trourent sur la même ver. ticale, ce qui a lieu lorsque l'on a :

$$
\frac{2}{3} e_{0}=\frac{1}{3} e \quad \text { d'où }: h=2 e_{0} \operatorname{cotg} \beta .
$$

En appliquant les formules élémentaires données au début de nos articles, on trouve facilement, pour $n^{\prime}, n^{\prime \prime}$ et $A$ en charge, les expressions simples suivantes, pour le cas où le niveau de l'eau affleure le niveau $\mathrm{CD}$ du couronne. ment.

$$
\begin{gathered}
n^{\prime}=y\left[K-\frac{y^{2}}{e^{2}}-\mu(\mu+K-2)+K n^{2} \frac{2-3 \mu-2 n}{1-\mu}\right] \\
n^{\prime \prime}=y\left[\frac{y^{2}}{e^{2}}+\mu(\mu+K-1)-K n^{2} \frac{1-3 \mu-2 n}{1-\mu}\right] \\
A=n{ }_{2}=n^{\prime \prime}\left(1+\operatorname{tg}^{2} \beta\right)
\end{gathered}
$$

On voit de suite qu'il y a avantage à réduire $\mu$ au strict nécessaire, car, à toute augmentation de $\mu$ correspond une augmentation de $n$ " et de $n^{\prime \prime}$, et une diminution de $n$ '.

L'augmentation de pression à l'amont, par rapport au triangle fondamental seul, est:

$$
\Delta\left(n^{\prime}\right)=K y \frac{n^{2}}{1-\mu}(2-3 \mu-2 n)
$$

Et la diminution correspondante à l'aval est :

$$
\Delta(n)=-K y \frac{n^{2}}{1-\mu}(1-3 \mu-2 \eta)
$$

La pression maxima maximorum est aussi diminuée de la quantité :

$$
\Delta\left(n_{2}{ }_{2}\right)=\Delta(n ")\left(1+\operatorname{tg}^{2} \beta\right)
$$

La courbe CDMB, de la figure 71 ci-jointe, rapportée à l'axe $O$ y $y$ représente la variation suivant les divers joints, du rapport $n^{\prime} / y$ que nous appelerons $\gamma$. Rapportée à l'axe O'y, distant du premier de la quantité $0 O^{\prime}$, qui représenterait la valeur constante du rapport $\gamma_{\mathrm{s}}$ du triangle fondamental s'il était seul, cette même courbe représente la variation de l'augmentation de y par rapport au triangle seul. Si l'on mène l'horizontale $B B^{\prime}$ ayant pour ordonnée le rapport ${ }_{11}$, qui correspond à la base $(y=H)$ du profil adopté, on voit que $\gamma$ n'est inférieur à $\eta_{1}$ que sur un très faible espace dans le voisinage immédiat du point $\mathrm{D}$, espace qui est d'autant plus faible que la valeur de $\mu$, et le rapport $n$ choisi pour la base, sont eux-mêmes plus taibles. (La courbe CMB correspond $\grave{a} n=0,03$ et $\mu=1 / 5$, et $\mathrm{OC}=K$ ).

$\Delta_{y}$ est nul pour $\eta=1-\frac{3}{2} \mu$ soit pour $y=2 y_{0} \frac{1-\mu}{2-3 \mu}$

Le maximum de $\Delta \gamma$ a lieu en $M$ pour $n=\frac{2}{3}-\mu$ qui annule la dérivée $\frac{\mathrm{d} \Delta \gamma}{\mathrm{d} n}$ soit pour $y_{m}=3 y_{0} \frac{1-\mu}{2-3 \mu}$ et ce maximum a pour valeur :

$$
\Delta_{m \gamma}=\frac{K}{1-\mu}\left(\frac{2}{3}-\mu\right)^{3}
$$

A cause de la faible hauteur du niveau de l'eau au-dessus du joint TD, et de la portion extrêmement restreinte du mur, pour laquelle cette infériorité se manifeste, on pourrait, à la rigueur, passer outre, puisque les conditions de stabilité seraient satisfaisantes sur tout le reste de la hauteur. Mais on peut y remédier très simplement en arrondissant l'angle en D. Pour cela, l'on remplacera la droite verticale $C D$ du parement aval du massif de couronne* 
ment, par une courbe cad convenablement choisie, de telle façon que les deux surfaces $a \mathrm{C} c$ et $a \mathrm{D} d$ soient égales et que de plus leurs centres de gravité soient à égale distance de la verticale $C D$. Si cette double condition est en effet remplie, rien ne sera changé dans les conditions de stabilité du barrage pour toute section horizontale située en dessous du point $d$, tandis que ces conditions de stabilité seront, au contraire, améliorées par toutes sections com. prises entre $a$ et $d$. Evidemment, cela entrainera une diminution de la pression $n$ ' pour la partie comprise entre $a$ et $c$, mais, comme cette pression y est notablement plus élevée que ce n'est nécessaire, il n'y a aucun inconvénient à cela; le remplacement de la ligne brisée $\mathrm{CD} d$ par la courbe cod a pour effet de régulariser les variations de la pression depuis le sommet jusqu'en $d$. Sur le graphique de la figure (71), la courbe CDM sera remplacée par la courbe CdM qui n'est plus rencontrée nulle part par la droite BB', qui correspond à la condition de stabilité désirée à la base.

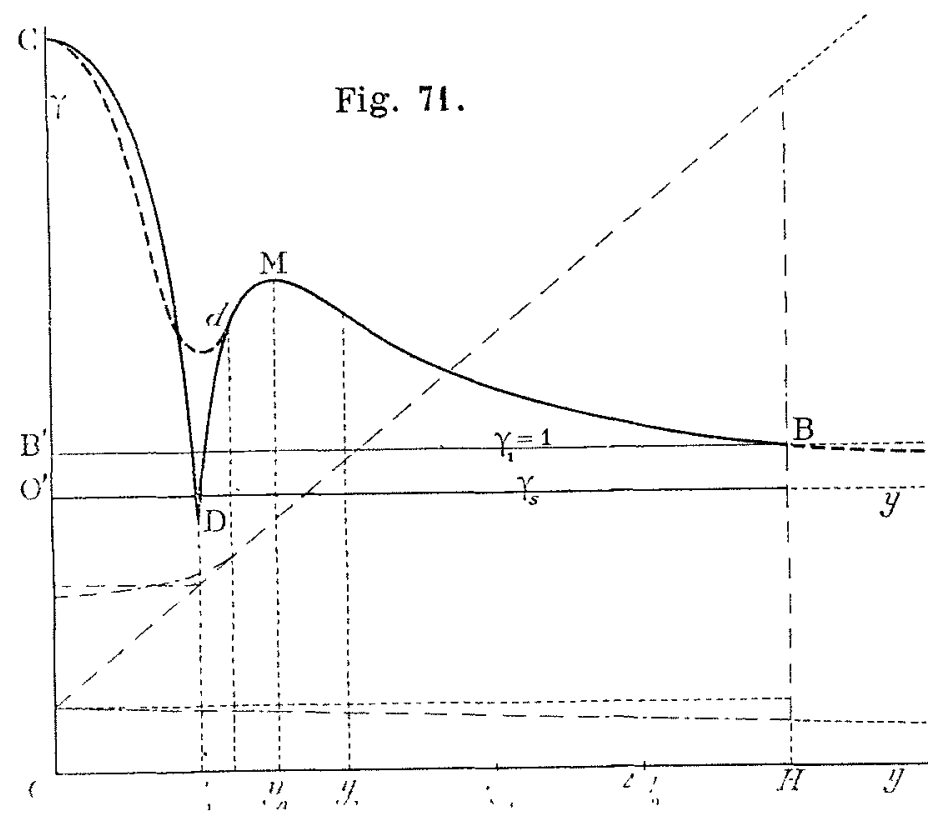

Théoriquement, il est toujours possible de tracer une courbe $c a d$, satisfaisant aux conditions précédentes; pratiquement, on pourra s'en rapprocher d'aussi près qu'on le voudra, d'ailleurs, une légère différence influerait relativement peu sur les conditions de stabilité à la base, les seules réellement importantes à considérer.

Si l'on a convenablement arrondi l'angle D, on peut être súr que la valeur de y calculée à la base est bien la plus petite valeur que ce rapport soit susceptible de prendre. Par suite, si la pression $n$ 'est supérieure à la base à celle de l'eau, elle le sera également sur toute la hauteur du mur.

De la relation (57) on peut en tirer l'expression suivante :

$$
e=\frac{y}{\sqrt{K-\gamma-\mu(\mu+K-2)+K \eta^{2} \frac{2-3 \mu-2 \eta}{1-\mu}}}
$$

qui donne immédiatement la largeur $e$ de la base $\mathrm{AB}$ pour des valeurs données de $y$, de $\gamma$, de $\mu$ et de $n$.

Les angles $\beta$ et $\beta_{1}$ d'inclinaison des parements seront donnés par les relations:

$$
\begin{aligned}
& \operatorname{tg} \beta_{1}=\mu \frac{e}{y} \\
& \text { à l'amont. } \\
& \operatorname{tg} \beta=(1-\mu) \frac{e}{y} \\
& \text { à l'aval. }
\end{aligned}
$$

Nous savons que $\mu$ doit être pris égal à 0,03 ; mais quelle valeur faut-il attribuer $\dot{a} n$. Plus $n$ sera grand, plus $e$ sera petit, et plus petite sera la surface du triangle SBA, mais plus grande aussi sera celle du triangle de couronnement SCD. Il y a donc une certaine valeur de $n$ qui doit rendre minima la surface totale. Or l'on a :

$$
S=\frac{e y}{2}\left[1+\frac{n^{2}}{1-\mu}\right]
$$

En portant dans cette expression la valeur de $e$, et en faisant $\frac{\mathrm{d} S}{\mathrm{~d}_{\mathrm{n}}}=0$, on arrive à l'équation :

$\eta^{3}-(2-3 \mu) \eta^{2}-3(1-\mu) \eta+\frac{1-\mu}{K}[2 \gamma-\mu(K+4-2 \mu)]=0$

En faisant $\mu=0,03$ et $\gamma=1$, on trouve $n=0,232$ pour $K=2,3$, et $n=0,265$ pour $K=2$.

C'est-à-dire que, tant que la hauteur de l'ouvrage n'est pas assez grande pour nécessiter une augmentation du fruit à l'amont, l'épaisseur du couronnement qui est la plus économique est voisine du quart de celle de la base.

Les courbes de la fig. 72, et le tableau ci-joint, montrent comment varient l'épaisseur à la base et la surface totale du profil, pour $\mu=0,03, \gamma=1$ et $K=2,3$, lorsque

\begin{tabular}{|c|c|c|c|c|c|c|c|c|}
\hline$n$ & 0 & $1 / 10$ & $1 / 8$ & $1 / 6$ & $1 / 5$ & 0,232 & $1 / 4$ & $1 / 3$ \\
\hline$\frac{e}{y}$ & 0,8804 & 0,8669 & 0,8601 & 0,8470 & 0,8353 & 0,8235 & 0,8168 & 0,7862 \\
\hline$\frac{S}{y^{2}}$ & 0,4402 & 0,4379 & 0,4370 & 0,4356 & 0,4349 & 0,4346 & $0,434 i$ & 0,4381 \\
\hline
\end{tabular}
l'on fait varier le rapport $n$. Il est bien entendu que ceci n'est applicable que si la hauteur de l'ouvrage reste inférieure à celle pour laquelle la pression à l'amont à vide atteint la limite pratique de résistance à imposer aux maçonneries.

Lorsqu'il s'agira d'établir un barrage au travers d'une gorge à section transversale sensiblement rectangulaire, il y aura avantage à donner au couronnement une largeur comprise entre un quart et un cinquième de la base, ou

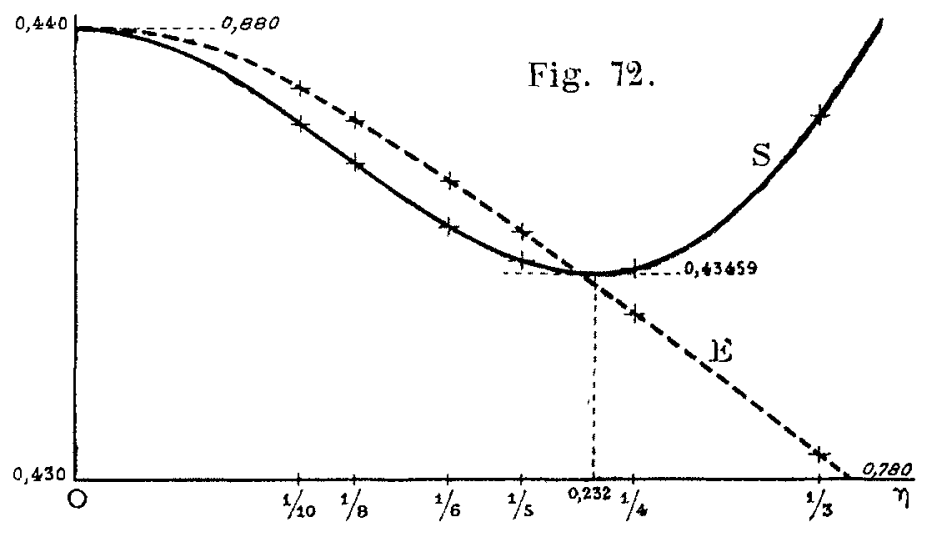

bien, ce qui revient au même, entre le cinquième et le sixième de la hauteur. On réalisera ainsi le profil á section minima, sans aucune complication pour la construction de l'ouvrage.

Dans le cas le plus général, ou la coupe transversale de la vallée affecte une forme quelconque, le minimum des maçonneries serait évidemment réalisé en attribuant le profil minimum aux diverses sections transversales correspondant aux diverses hauteurs du mur, c'est-à-dire en faisant varier la largeur du couronnement proportionnelle- 
ment à la hauteur; mais ceci n'est pratiquement réalisable que dans des limites assez restreintes. En effet, on ne peut songer à donner nulle part au mur une épaisseur inférieure à $2 \mathrm{~m}$., et, de plus, si les variations de largeur du couronnement était trop grandes, le barrage, vu d"un endroit plus élevé, se présenterait en plan sous une forme quelque peu disgracieuse.

Jusqu'ici, du moins pour les barrages que nous connaissons, on a maintenu l'épaisseur $e_{0}$ constante sur toute la longueur de l'ouvrage. Avec ce système, le rapport $n$ doit être inférieur à un cinquième pour le profil de plus grande hauteur, et cela d'autant plus que la coupe transversale de la vallée se rapproche plus du triangle que du rectangle. Quant à la recherche de la meilleure valeur de $e_{0}$, elle serait fort laborieuse, aussi cette largeur se détermine-t-elle au jugé. (Dans son ouvrage sur les barrages en maconneries, M. KRANTz avait proposé d'adopter pour le couronnement uné largeur sensiblement égale au dixièms de la hauteur de la retenue (*), et, après lui, celte proposition a été plusieurs fois renouvelée et appliquée). Or, en général, les barrages sont utilisés comme moyen de communication entre les deux cotés de la vallée, et le couronnement supporte une route dont la largeur est déterminée par des considératıons de voirie. Cette largeur est parfois supérieure à celle que lon est amené à donner à $e_{0}$, aussi, bien souvent, la route repose-t-elle en encorbellement à l'aval sur une série de voutes, de profondeur constante, qui s'appuient au moyen de pilastres sur le parement aval.

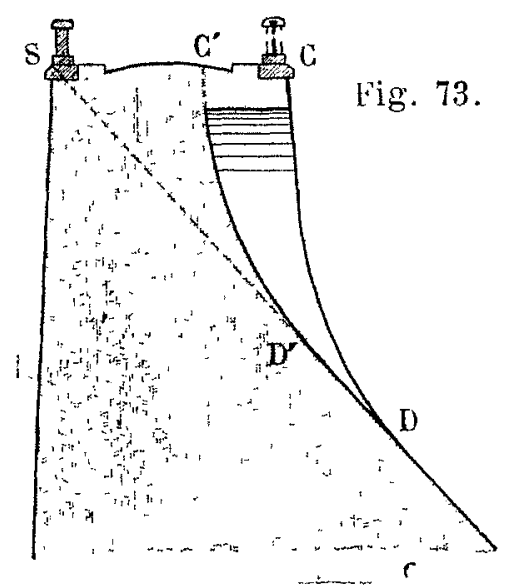

L'emploi de ces voûtes conduit à une nourelle soIution du problème. Cette solution, qui nous paraît la plus rationnelle, consiste à composer le couronnement en partie d'une partie pleine SC'D', d'épaisseur variable, et en partie d'une série de voùtes $\mathrm{DCC}^{\prime} \mathrm{D}^{\circ}$ dont la profondeur CC' serait également variable. Réduite dans les parties les plus hautes à un minimum 0 n20 à $0 \mathrm{~m} 30$, pour assurer la continuité de l'aspect, cette profondeur irait en croissant jusqu'à ce que la partie pleine SC'D' soit réduite à 2 ou 3 mètres. Lorsque la hauteur maxima du barrage se trouvera sensiblement vers le milieu de la vallée, on pourra même faire varier légèrement la largeur totale $\mathrm{SC}$, ainsi que nous le montrerons plus loin dans un exemple. Le couronnement présentera ainsi un lèger renflement vers son milieu, assez petit pour ne pas être désagréable à l'œil $\left(^{\star \star}\right)$, mais suffisant cependant pour faciliter les moyens de communications, en permettant à deux voitures de se croiser au milieu de l'ouvrage, ce qui serait matériellement impossible sur beaucoup de barrages actuels. Nous n'insisterons pas davantage sur ce dispositif, toutefois, nous ferons remarquer que, quelque

(") Une largeur de couronnement égale au dixicme de la hauteur est aussı sensiblementégale au neuvième de cette mème hauteur (pour $n^{\prime}=y$ ). Or, li surface correspondante est à peu près égale à 0,43745 , de sorte que l'augmentation de surface, par rapport à celle qu correspond au profil minimum, est de 0, e5 pour 100 .

(*) On pourra maintenir le point $D$ à une profondenr sensiblement constante, tout en fassant varter la largeur $\mathrm{CD}$ du couronnement. Il suffira pour cela de faire varier un peu linclinasson de GD, surtout tans la partie cintrée du bas. soit la valeur que l'on soit amené à donner à $e_{0}$, l'emploi des voutes permet de rompre la monotonie de la construction, et de donner un certain aspect architectural à l'ouvrage, ce qui n'est pas à dédaigner, trop de gens étant portés à dénigrer systématiquement les entreprises des ingénieurs, sous le prétendu prétexte qu'ils déparent la beauté des sites les plus pittoresques !

Le profil pratique triangulaire à parements rectilignes, que nous venons d'étudier, est bien certainementle plus simple qu'il soit possible d'imaginer, tant au point de vue des facilités de la construction, qu'à celui de la simpplicité des calculs auxquels il conduit, à tel point qu'on peut déterminer immédiatement les dimensions à donner à un barrage pour réaliser certaines conditions données, sans avoir à s'astreindre à toute une série de laborieux calculs, comme cela a lieu pour des profils quelconques; aussi l'avons-nous étudié d'une manière toute spéciale. Mais l'on peut imaginer d'autres types pratiques dérivant du profil triangulaire, et notamment le suivant, qui peut procurer une très légère économie dans le cube des maconneries par rapport au type précédent.

$\mathrm{Au}$ lieu de donner au parement amont une légèrc inclinaison, qui demeure constante sur toute la hauteur du mur, on peut maintenir le parement amont d'abord vertical sur une certaine hauteur $h_{1}$, correspondant à celle pour laquelle la verticale du centre de gravité du massif vient passer juste par le tiers du joint EE', c'est-à-dire sur une hauteur égale au double de celle $\mathrm{CD}$ du triangle de couronnement.L'influence de ce couronnement faisant alors pénétrer la verticale du centre de gravité dans le tiers amont, il faut donner au parement amont une certaine inclinaison, et cela sur une certaine hauteur $h_{2}$; puis l'on ramène le parement à être vertical sur le reste $h_{3}$ de la hauteur du mur.

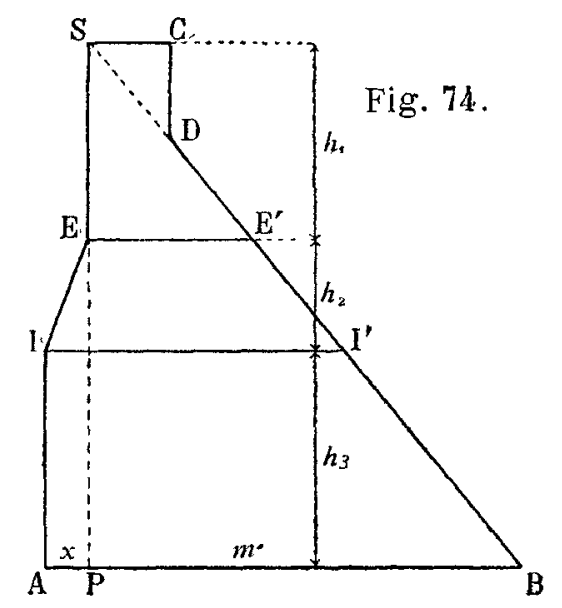

Désignons, comme précédemment, par $e_{\mathrm{o}}$ la largeur du couronnement. et par $y_{0}$ la hauteur du triangle auxiliaire. Désignons également par $H$ la hauteur totale du mur, par $\beta$ l'angle du parement aval avec la verticale, par $\beta_{1}$ l'angle que fait, avec cette même verticale, l'élément EI du parement amont, par $x$ la projeclion AP encore inconnue de l'élément EI sur l'horizontale, et par $m$ ' la projection du parement aval sur cette même horizontale. En calculant la distance au point A de la verticale du centre de gravité du profil AIESCDB, et en écrivant que cette distance est égale au tiers de la base $\mathrm{AB}$, on arrive à l'équation 61 suivante :

$\left(h_{2}+h_{3}\right) x^{2}+2\left[e_{0} y_{0}+m^{\prime}\left(2 y_{0}+\frac{h_{2}}{2}\right)\right] x-\left(h_{2}+h_{3}\right) e_{0}^{2}=0$

Liangle $\beta_{1}$ d'inclinaison de l'élément EI se déterminera par la condition que, pour aucune autre section comprise entre $E$ et I, la verticale du centre de gravité du massif situé au-dessus de cette section ne vienne à pénétrer dans le tiers amont du joint. Or, si dans l'équation précédente on fait $h^{3}=0$, si l'on y cousidère $h_{\mathrm{g}}$ et $m$ ' comme des variables, 
et si l'on y remplace $x$ par $h_{2} \operatorname{tg} \beta_{1}$, on obtient la nouvelle écuation 62 :

$l_{2}^{2} \operatorname{tg}^{2} \beta_{1}+2\left[e_{0} y_{0}+\left(2 y_{0}+l_{2}\right)\left(2_{y_{0}}+\frac{h_{2}}{2}\right) \operatorname{tg} \rho\right] \operatorname{tg} \beta_{1}-c_{0}^{2}=0$ qui exprime précisément la condition que, pour chaque section, la verticale du centre de gravité passe par le tiers du joint considéré

En attribuant à la hauteur $h_{2}$ toutes les valeurs qu'elle est susceptible de prendre cntre $\mathrm{E}$ et $\mathrm{I}$, on obliendra diverses valeurs de tgi $\beta_{1}$, et, en adoptant la plus grande de toutes, on sera bien sûr que l'on n'aura nulle part à craindre des cfforts d'extension à l'aval à vide, or, il est facile de voir que la valeur de tg $\beta_{1}$ diminue au fur et à mesure que $h_{g}$ ausmente, c'est-à-dire que la valeur maxima de tg $\beta_{1}$ a lieu pour $h_{2}=0\left(^{*}\right)$, Si donc l'on fait $h_{2}=0$ dans l'équation précédente (62), il vient :

$$
\begin{array}{r}
2\left(e_{0} y_{0}+4 y_{0}{ }^{2} \operatorname{tg} \beta\right) \operatorname{tg} \beta_{1}=e_{0}{ }^{2} \\
\text { ce qui donne finalement : } \operatorname{tg} \beta_{1}=\frac{1}{10} \operatorname{tg} \beta
\end{array}
$$

En portant cette valeur de $\operatorname{tg} \beta_{1}$ dans l'équation (61), celle-ci deviendra :

$$
\left.\begin{array}{c}
\frac{2}{10}\left[e_{0} y_{0}+\left(H-2 y_{0}\right)\left(2 y_{0}+\frac{h_{0}}{2}\right) \operatorname{tg} \beta\right] h_{2} \operatorname{tg} \beta \\
+\frac{H-2 y_{0}}{100} h_{2}{ }^{2} \operatorname{tg} \beta-\left(H-2 y_{0}\right) e_{0}{ }^{2}
\end{array}\right\}=0
$$

équation lu second degré dont on tirerait la valeur de $h_{a}$, si $\operatorname{tg} \beta$ étalt connu. La hauteur $h_{2}$ étant déterminée, on en déduirait immédiatement la valeur de $x$.

En écrivant que la pression élastique normale $n$ 'à l'amont en charge est égale à celle de l'eau, on obtiendrait une nouvelle équation de condition qui, jointe à la précédente, parmettrait de déterminer tgis. Mais l'expression ainsi trouvée pour tg $\beta$ serait très compliquée, et il pourra être aussi avantageux de calculer to $\beta$ comme s'il s'agissait du premier profil, ct de modifier approximativement le résultat trouvé.

On verra plus loin, par deux exemples, que, pour un même cube de maçonnerie, ce dernier profil peut procurer une légère augmentation de la stabilité par rapport au profil précédemment étudié. Ceci peut, d'ailleurs, se.prévoir a priori, puisque, d'une part, le parement amont est moins incliné, et que, d'autre part, la verticale du centre de gravité du mur peut passer exactement par le tiers de la base.

Nous n'insisterons pas davantage sur ce type de profil, toutefois, nous ferons encore remarquer que la pression limite qu'on s'est fixée sera atteinte à vide avec une hauteur moindre pour ce profil que pour le précédent.

La pression normale à l'amont a pour valeur à vide:

$$
n_{0}^{\prime}=K^{\prime} y\left(1-\mu+n^{2} \frac{2-3 \mu-20}{1-\mu}\right)
$$

$K^{\prime}$ étant la densité réclle des maconneries. Cette définition de $K^{\prime}$ nécessite une explication. Le Ve Congrès de navigation intérieure, tenu à Paris en 1892 , a estimé qu'il était prudent, pour le calcul des barrages en charge, d'attribuep à la maçonnerie un poids inférieur de $100 \mathrm{kgs} \mathrm{par} \mathrm{m}^{3}$ à son poids réel; ceci, afin de tenil compte du délayement et de l'appauvrissement des mortiers causés à la longue par les infiltrations, et de se fixer en même temps un léger coefficient de sécurilé, car, plus la densité est faible, plus

\footnotetext{
(1) La valcur de $\frac{d \operatorname{tg} \beta_{1}}{d h_{2}}$ est toujours négative, par suite $\operatorname{tg} \hat{i}_{1}$ diminue lorscue $h_{2}$ augmente.
}

grandes doivent être les dimensions des ouvrages. Comme un excès de prudence ne nuit jamais en pareille matière, nous adopterons cette manière de voir. D'ailleurs, la circulaire du Ministère de l'Agriculture du 15 juin 1897, déjá citée, exige que les calculs d'établissement des barrages satisfassont a cette condition. Mais, à vide, la maçonnerie doit être calculée avec son poids réel. La densité $K$ qui entre dans le calcul des pressions en charge dort donc être prise égale à $\left(K^{\prime}-0,1\right)$ : par exemple $K^{\prime}=2,4$ et $K=2,3$.

L'augmentation $\Delta\left(n_{0}^{\prime}\right)$ de pression à l'amont à vide par rapport au triangle simple, est $\frac{K^{\prime}}{K} \Delta\left(n^{\prime}\right)$. Par suite, à toute augmentation, ou diminution de pression, en charge à l'amont. correspond sensiblement une égale augmentation - ou diminution - de pression à vide. Rigoureusement, la compression maxima à vide $A_{0}$ est égale à $n_{0}\left(1+\operatorname{tg}^{2} \beta_{1}\right), \beta_{1}$ étant l'angle d'inclinaison du parement amont sur la verticale. Mais, en pratique, $\beta_{1}$ doit être assez petit, de sorte que l'on peut confondle $A_{0}$ avec $n_{0}^{\prime}$ du moins pour une première approximation. Lorsque la résistance limite $\rho$ est alleinte, il faut augmenter la valeur de $\mu$, ou diminuer celle de $e_{0}$, de manière à rapprocher la verticale du centre de gravité du mur près du milieu de la base, afin de diminuer $n{ }_{0}^{\prime}$.

Si l'on fail $K^{\prime}=2,4, \mu=0, \bar{u} 8, \gamma=1$ et $\rho=120$ tonnes par $\mathrm{m}^{2}$, la hauteur maxima à laquelle on puisse monter, sans dépasser cette limite $\rho$, est de $47 \mathrm{~m}$. pour $n=1 / 4$, et de $50 \mathrm{~m}$. pour $n=1 / 8$. (Pour $n=0$ cette hauteur sorait de $51^{\mathrm{m}} 50$ ).

Si l'on portait la limite $\rho$ jusqu'à 160 tonnes par $\mathrm{m}^{2}$, les hauteurs précédentes seraient reculées respectivement jusqu'à $63 \mathrm{~m}$., $67 \mathrm{~m}$. et $69 \mathrm{~m}$.

Nous venons de voir que tant que la hauteur était inférieure à $47 \mathrm{~m}$. (pour $K^{\prime}=2,4$ et $\rho=120$ ), la surface du profil était minima lorsque $n$ était compris entre $1 / 4$ et $1 / 5$, mais lorsque la hauteur devient plus grande, il faut augmenter $\mu$, et alors la valeur la plus avantageuse de $n$ décroit rapidement : c'est ainsi que pour $50 \mathrm{~m}$. ellc n'est plus que $1 / 7$, et pour $60 \mathrm{~m}$. ce serait théoriquement 0 . Pour les très grandes hauteurs, la largeur $c_{0}$ doit être donc réduite au strict minimum.

La valeur qu'il faut donner à pour que la pression maxima $A_{0}$ à vide no dépasse pas la limite $\rho$ qu'on s'est fixce, s'obtiendrait en tirant la valeur de $e$ de l'équation :

$$
A_{0}=n_{0}^{\prime}\left(1+\mu^{2} \frac{e^{q}}{y^{9}}\right)=\rho
$$

et en la portant dans l'équation (59).

Mais l'on serait ainsi conduit à la résolution d'une équation du $4^{\text {e }}$ degré en $\mu$, et si l'on veut s'éviter la résolution te cette équation, on pourra employer la méthode des approximations successives.

Dans une première approximation, on remplacera $A_{0}$ par $n_{0}^{\prime}$, ces deux valeurs différant relativement peu l'une de l'autre, el l'on tirera la valeur de $\mu$ de l'équation (65); on aura ainsi une première valeur approchée $\mu_{1}$ en prenant la plus petite des deux racines de l'équation :

$$
\mu^{2}-\left(2+3 \eta^{2}-\frac{p}{K^{2} y}\right) \mu+1+2 \eta^{2}(1-\eta)-\frac{p}{K^{\prime} y}=0
$$

On obtiendra ensuite une première valeur approchée $e_{1}$ de la base en portant la valeur de $\mu_{1}$ ainsi trouvée dans la relation (59), ou bien encore dans la suivante:

$$
\frac{e}{y}=\sqrt{\frac{K}{K^{\prime}} \frac{p}{y}-\gamma+\mu(2-\mu)}
$$


qui est plus simple, et qu'on obtient immédiatement en remarquant que les équations (57) et (65) peuvent s'écrire :

$$
\begin{gathered}
\gamma=K-\frac{y^{2}}{e^{2}}-\mu(\mu+K-2)+\frac{\Delta\left(n^{\prime}\right)}{y} \\
\frac{\rho}{y}=K^{\prime}(1-\mu)+\frac{K^{\prime}}{K} \frac{\Delta\left(n^{\prime}\right)}{y}
\end{gathered}
$$

On obtiendra une nouvelle valeur $\mu_{2}$, plus approchée que la précédente, en portant les valeurs de $\mu_{1}$ et de $e_{1}$, ainsi obtenues, dans le terme $\Delta_{0}$ et dans celui en $\mu^{2}$ de l'expression de $A_{0}$ mise sous la forme :

$$
A_{0}=\left[K y(1-\mu)+\Delta_{0}\right]\left(1+\mu^{2} \frac{e^{2}}{y^{\underline{a}}}\right)
$$

On aura alors une équation du premier degré à une inconnue dont on tirera la valeur de $\mu_{2}$, puis l'on déterminera ensuite la valeur de $e$ en portant dans l'équation (59) la valeur de $\mu_{2}$ ainsi obtenue.

Pour atténuer la compression maxima en charge, qui levient considérable lorsque la hauteur est très grande, on peut diminuer linclinaison du parement aval dans le voisinage de la base en accolant à ce parement un massif supplémentaire de maçonnerie, et en donnant au profil une forme qui ressemble à ceux de MM.Guillemain et Hetier, tout comme pour le profil suivant représenté par la figure 75 . Toutefois, l'on doit agir avec prudence, car si le terme tge $\beta$ diminue rapidement avec $\beta$, la pression normale $n$ " augmente par contre, puisque l'adjonction du massif supplé. mentaire MNB a pour effet de déplacer vers l'aval le contre de gravité de l'ensemble du système.

$\mathrm{Au}$ lieu de donner au parement amont une inclinaison uniforme, on peut aussi l'établir, tout d'abord, sensiblement vertical $(\mu=0,03)$ sur une certaine hauteur $h$, puis

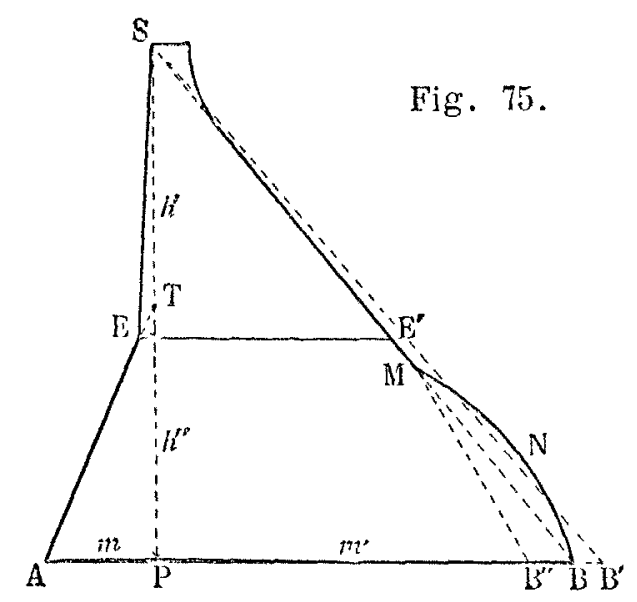
augmenter ensuite linclinaison sur le restant $h "$ de la hauteur, et cela de la quantité voulue, de manière à ne jamais dépasser la pression limitequ'on s'est fixée $\dot{a}$ vide. A laval, pour diminuer la valeur de la pression en charge, on redressera le parement en accolant au triangle fondamental, un massif auxiliaire MNB. On réalisera ainsi le profil SEABNMS, représenté sur la fig. 75 ci-jointe.

Il n'est plus possible, dans ce cas, davoir des relations simples, permettant de déduire immédiatement la valeur de la base ainsi que celles des diverses inclinaisons; mais, cependant, il est possible d'obtenir des valeurs suffisam. ment approchées pour éviler de longs tâtonnements.

Comme le couronnement doit' être réduit au strict minimum, et que, d'autre part, les surfaces SET et BNM sont rclativement faibles par rapport à la surface totale du profil, on peut, sans grande erreur, et du moins pour une première approximation, remplacer le profil réel par un profil triangulaire fictif, composé de deux triangles rectangles SPB' ct TAP, de hauteur's inégales, et accolés l'un à l'autro.

Soit $H$ la hauteur du mur, et désigrions respectivement par $m$ et par m" Ios projections AP et PB' des parements
TA et SB'. Le point $T$ peut, sans grande erreur, être confondu avec le point d'interseclion de SP et de EE', de sorte qu'il vient, pour la valcur en A de la pression n'。 à vide :

$$
n_{0}=K \frac{H m^{\prime \prime}-\left(H-2 h^{\prime \prime}\right) m " m}{(m "+m)^{2}}
$$$$
\text { de mème: } \quad A_{0}=n_{0}^{\prime}\left(\frac{h^{\prime \prime Q}+m^{2}}{h^{\prime \prime 2}}\right)
$$

En développant cette dernière équation, et en écrivant que $A_{0}$ doit être égal à la pression limite $\rho$, on arrive à l'équation du $3^{c}$ degré :

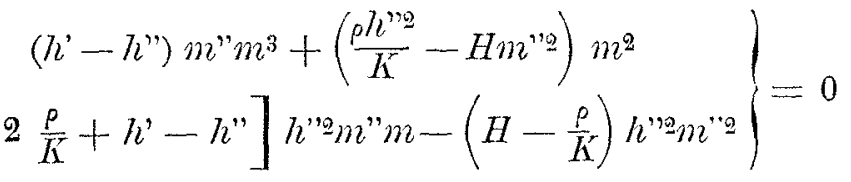

Nous admettrons que l'on s'est donné a priori les valeurs de $h$ et $h$ ". Your avoir une valeur approchée de m", on admettra que le triangle SPB' est équivalent au triangle $\mathrm{SA}^{\prime} \mathrm{B}\left(^{*}\right)$, et on calculera la largeur m" de sa base comme s'il s'agissait du profil précédent à parements rectilignes on se contentant de la promière approximation.

Dans certains cas particuliers, surtout lorsque la hauteur du mur sera considérable, les hauteurs $h$ ' et $h$ " pourront différer assez peu l'une de l'autre pour que l'on puisse négliger les termes en ( $\left.h^{\prime}-h^{\prime \prime}\right)$. Dans ce cas, l'équation (70) se réduira au second degré, ce qui simplifiera les calculs.

Pour déterminer tg $\beta$, on cherchera pour le parement aval une inclinaison telle, que la surface du profil soit à peu près la même que celle du profil précédent à parements rectilignes. On vérifiera toutefois que la valeur du rapport $e / y$, auquel on sera conduit sur le joint EF', est au moins égale à celle du tableau de la page 280 pour la valeur correspondante de $n$, sinon lon aurait sur ce joint $n<y$.

lin général, on ne tombera pas du premier coup sur le résultat cherché, mais l'écart ne saurait être considérable, et, en modifiant légèrement, en plus ou en moins, les premières dimensions adoptées, on arrivera rapidement à déterminer le profil lo plus convenable. Un exemple montrera plus loin comment l'on peut employer la méthode précédente.

Dans ce qui précède, nous avons supposé que le niveau maximum de l'eau affleurait le summet du mur, niveau correspondant à celui des plus fortes crues, augmenté d'une certaine hauteur pour tenir compte de l'effet des vagues. Or, l'on admet généralement que cette hauteur doit être comptée comme égale seulement à la rnoitié de l'amplitude des vagues; par suite, si la retenue ainsi comptée afficure le sommet du mur, le parapet d'amont de Ia roule, que supporte en général tout barrage, aura donc à résister au choc des vagues et surtout des corps flottants. Aussi, bien souvent, admet-on une légère surélévation du mur au-dessus de la retenue prévue, ce qui a, en outre, l'avantage d empêcher l'eau de se déverser par dessus le barrage en cas de crue tout à fait exceptionnelle.

Si, dans les formules que nous avons précédemment établies, on admet que la pression n' soit jusle égale à celle de l'eau lorsque le niveau de celle-ci est supposée atteindre le sommet du nuur, cotte pression n' lui sera superieure si la retenue maxima prévue reste en dessous du sommet $S$.

(') Le point $\mathrm{A}^{\prime}$, omis par erreur sur la figure 75 , doit se trouver à l'intersection de la base $A B$ avec le parement $S E$ prolongé. 
Comme il vaut mieux pécher par excès de sécurité, on devra donc calculer les dimensions d'un barrage comme si la hauteur de l'eau atteignait le couronnement, et régler le déversoir de telle facon que, en cas de crues, le niveau maximum de l'eau reste inférieur au couronnement.

Applications, - A titre d'exemple, nous allons indiquer comment on applique la théoric précédente au calcul d'un barrage à profil triangulaire.

Soit à calculer un barrage triangulaire à parements rectilignes, devant créer une retenue maxima de $45 \mathrm{~m}$. de hauteur au-dessus du sol, y compris la surélévation correspondant au mouvement des vagues, et au déversement des eaux par dessus le déversoir en temps de fortes crues.

Nous admettrons, a prior $i, n=1 / 6, \mu=0,03$ et $K=2,3$, dès lors, la formule (59) nous donne immédiatement $e=0,847 y=38 \mathrm{~m} 115$. La largeur correspondante $e_{0} \mathrm{du}$ couronnement pour la hauteur maxima sera donc $e_{0}=6^{\mathrm{m}} 352$.

Quand à l'inclinaison des parements, la formule (60) nous donne $\operatorname{tg} \beta_{1}=0,0254$ pour celui d'amont, et $\operatorname{tg} \beta_{2}=0,8216$ pour celui d'aval.

Nous adopterons $e_{0}=6{ }^{\mathrm{m}} 35, \operatorname{tg} \beta_{1}=0,025, \operatorname{tg} \beta_{2}=0,822$.

Les dimensions du mur seront done, pour le profil correspondant au maximum de hauteur :

$$
\begin{gathered}
e_{0}=6^{\mathrm{m} 35} \quad m=\ln ^{\mathrm{m} 125} \quad m^{\prime}=36^{\mathrm{m} 99} \quad e=38^{\mathrm{m} 115} \\
n=0,166 \quad \mu=0,0295 \quad S=88^{\mathrm{m} 2} 1145
\end{gathered}
$$

Si l'on vérifie les conditions de stabilité à la base, on trouve :

$$
n^{\prime}=451008 \quad n^{\prime \prime}=62780 \quad A=1071820 \quad A_{0}=1061480
$$

$\mathrm{Au}$ fur et à mesure que l'on se rapprochera des flancs de la vallée, on diminuera la largeur du couronnement jusqu'à un minimum de $2 \mathrm{~m}$. environ. Une série de voûtes, dont la profondeur ira graduellement en s'augmentant, servira à la fois à supporter une route établie sur le couronnement (dont la largeur minima aux extrémités, parapets compris, sera comprise entre $4 \mathrm{~m}$. et $4^{\mathrm{m}} 60$ ) et à donner à l'ouvrage un certain aspect architectural $\left(^{*}\right)$. La largeur de $6^{\mathrm{m}} 35$ indiquée est une largeur virtuelle, qui correspond au massif plein fictif de couronnement, équivalent au volume réel, déduction faite du vide des voûtes. Suivant l'état des lieux et la forme des voûtes employées, la largeur réelle varicra, mais elle sera toujours supérieure à la largeur virtuelle.

Supposons maintenant que l'on veuille adopter le profil à parement amont discontinu de la figure 74 .

(c) Sur la figure 76 ch-jounte, la ligne en gros trats pointillós coriespond and diverses positions du point $G$ de la figure 73 . Cette figure 76 a été étahlie en supposant que la largeur de la vallée était de $100 \mathrm{~m}$. au niveau du couronnement, et lo 20 a la base, la coupe transversale de la vallée se rapprochant plus du trangle gue du rechangle. Afin d'avoir sur cetle figure des dmensions transversalos qui ne solent pas trop réduites, nous avons allopté pour ces dimensions une echelle double de celles des dımensıons longludinales (la flèche, prise égale au vingtième de la largeur de la valléc au sommet, a été doublée aussi). Ja largeur du couronnement est de $6^{\mathrm{m}} 35$ au milien ot se ridult à fin $^{\prime \prime} 50$ au extrémites, En ces pounts, l'ćpasseur $\mathrm{S} G$ du massif plon est réduite à $2^{\mathrm{m}} 25$.

Nэтa. - En étudiant l'influence du couronnement sur les conditions dequilibre d'un barrage établi suivant le profil triangulairc, nous avons été amení à montrer lo parti que l'on poturrait tirer dans certans cas d'un couronnement de largeur variable. Mais nous tenons à bien spécitier yue nous n'entendons nullemenl le précomser d'une mantère absoluc. Dans certans cas, surtout pour des vallees a scchon transversale triangulaire, le supplément de dépenses occasionné par la modification à apporter aux voûtes pourra compenser la petite bconomie sur les maçonneries róalisée par l'emplos du profil iummum.
Nous calculerons au préalable les dimensions du mur comme s'il s'agissait du profil précédent à parements rectilignes. Ceci fait, nous adopterons la même laroeur de cou. ronnement $e_{0}$, puis, nous modifierons au jugé l'inclinaison du parement aval. Comme la projection horizontale AP du parement amont doit être plus faible avec ce profil qu'avec le précédent, nous augmenterons legèrement $\operatorname{tg} \beta$, de manière à avoir sensiblement le même cube de maçonneries.

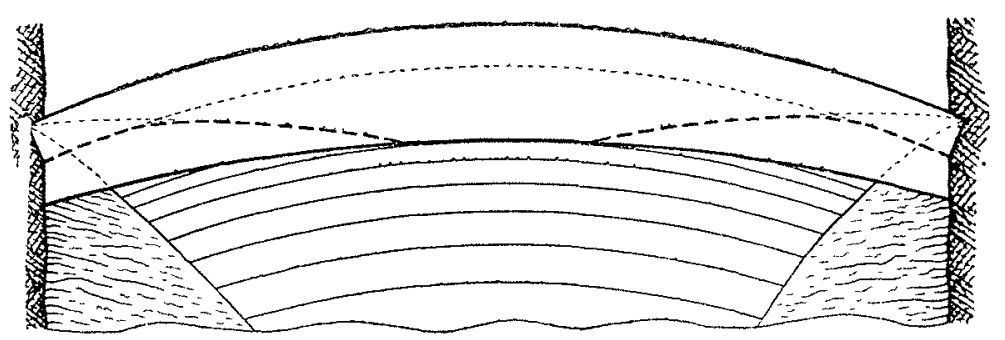

Fig. 76.

Nous ferons done, pour une première approximation $\operatorname{tg} \beta=0,83$, ce qui nous donnera immédiatement :

$$
e_{0}=6^{\mathrm{m} 35} \quad h_{1}=2 y_{0}=15^{\mathrm{m}} 30 \quad m^{\prime}=37^{\mathrm{m}} 35
$$

L'application des formules (63) et (64) nous donne en outre : $\operatorname{tg} \beta_{1}=0,083, h_{2}=9^{\mathrm{m} 02}, x=0^{\mathrm{m} 749}$.

On en déduit ensuite :

$$
h_{3}=20^{\mathrm{m}} 68 \quad e=38^{\mathrm{m}} 099 \quad S_{1}=883,531 \mathrm{~m}^{2} .
$$

Comme vérification, l'on trouve à la base :

$n_{1}=45448 \quad n^{\prime \prime}{ }_{1}=621007 \quad A_{1}=1041723 \quad A_{01}=111314$.

La valeur de $n_{1}$ est plus grande que celle de l'eau; $A$ ct $A_{01}$ sont inférieures à $12 \mathrm{kgs}$ par $\mathrm{cm}^{2}$; les conditions de stabilité sont donc excellentes, toutefois, $n$ ' pourrait être légèrement réduit sans inconvénient, puisque les $45 \mathrm{~m}$. de la retenue correspondent au cas le plus défavorable.

D'ailleurs, à titre de comparaison avec le profil précédent, nous allon's réduire encore un peu tg. et lui attribuer la valeur 0,828 , ce qui nous donnera :

$$
\begin{aligned}
& \operatorname{tg} \beta_{1}=0,083 \quad h_{1}=15^{\mathrm{m} 3 \mathrm{l}^{\prime}} \quad h_{2}=9^{\mathrm{m} 03} \quad m^{\prime}=37^{\mathrm{m} 26} \\
& x=0 \mathrm{~m} 750 \quad h_{3}=20^{\mathrm{m}} 63 \quad e=38^{\mathrm{m}} 01 \quad S_{\mathrm{q}}=881,558 \mathrm{~m}^{2} \\
& n_{2}^{\prime}=45_{1} 56 \quad u_{2}^{\prime}=621315 \quad A_{2}=105036 \quad A_{02}=111^{1} 326
\end{aligned}
$$

Sur le joint EE' on trouve que la pression $n$ ' a l'amont est égale à 281250 , alors que la pression de l'eau n'y est que de 241370 .

La valeur de $n_{2}^{\prime}$ est plus forte encore que celle $n$ ' du profil à parement amont rectiligne; toutefois, la différence entre $n_{2}^{\prime}$ et $n^{\prime}$ n'est plus que la moitié de ce qu'elle était entre $n_{1}^{\prime}$ et $n^{\prime}$, de sorte que le profil équivalent de ce type doit avoir son parement aval sensiblement incliné à 0 m827 par'mètre.

Dans le cas le plus général oú deux approximations ne conduiraient pas au résultat cherché, on déterminera la valeur de $\operatorname{tg} \beta$ par interpolation.

Si l'on compare les deux séries de valeurs trouvées dans les approximations précédentes pour le profil à paroment amont discontinu, avec celles du profil a parements rectilignes, on voit que la surface $S$ de ce dernier est comprise entre $S_{1}$ et $S_{2}$, tandis que sa pression $n$ ' est encore inférieure à $n_{2}$. En procédant par interpolation, on trouverait que le profil qui donnerait $n_{3}^{\prime}=n^{\prime}=45^{1008}$, aurait une section sensiblement égale à $880,560 \mathrm{~m}^{2}$, ce qui conduit à une diminution de 0,18 pour 100 dans le cube des maçonneries. 
On voit donc que ce profil peut permettre une très légère ćconomie dans le cube des maconneries, par rapport au profil à parements rectilignes. Mais, alors que pour ce dernier il sulfit de faire varier la largeur du couronnement pour réaliser partout lo profil minimum, il faudrait ici faire varier en même temps $h_{1}, h_{9}, h_{3}$ et $x$, ce qui aurait pour inconvénient de compliquer la construction et d'entrainer un supplément de dépense. Si l'on maintier fixes ces dernières dimensions, tout en faisant varier $e_{0}$, ce profil peut devenir moins économique que l'autre. Il n'est donc réellement avantageux que si la coupe transversale de la vallée est sensiblement rectangulaire, ce qui est d'ailleurs assez rare, ou bien si l'on maintient constante la largeur du couronnement.

Soit maintenant à calculer un barrage à parements rectilignes de $70 \mathrm{~m}$. de hauteur, en s'imposant la condition que la pression maxima ne soit jamais supérieure à $14 \mathrm{kgs}$ par $\mathrm{cm}^{2}$, la densité r'ćelle $K$ ' des maçonneries étant prise égale à 2,4 .

Nous avons vu quen devail ĉtre réduit au strict minimum, or, on ne peut guère descendre à moins de $4 \mathrm{~m}$. de largeur en couronne pour le profil de hauteur maxima, d'autre part, pour $n=0$, la largeur de la base devrait être d'au moins $62 \mathrm{~m}$, on peut donc poser $n=1 / 16$. Dans ce cas, la résolution de l'équation (67) nous donne, pour une première approximation, $\mu_{1}=0,173$, et la valeur correspondante de $e$, tirée de la relation (68) est $e=0,90 y$; de sorte que l'équation (69) devient:

$$
1,0243[168(1-\mu)+0,0063]=140
$$

d'où l'on tire $\mu=0,193$.

En portant cette valeur de $\mu$ dans la relation (59), celle ci donne $e=0,9079 y$. Pour diminuer la pression maxima à l'aval, nous allons redresser le parement $a$ la base en accolant à ce parement un segment de cercle tel que sa tangente en $B$ fasse un angle de $10^{\circ}$ avec la direction du parement du triangle fondamental. Mais ceci ayant pour effet de déplacer vers l'aval le centre de gravité du profil, la pression $n$ ' à l'amont se trouvera diminuée; il nous faut donc augmenter légèrement la valeur de $e$ ainsi trouvée, aussi prendrons nous $e=0,92 y$; nous diminuerons aussi très légèrement la valeur de $\mu$ que nous ferons égal à 0,19 . Nous aurons alors $e=64^{\mathrm{m}} 40$, et nous donnerons au couromement une largeur de 4 mètres.

Les inclinaisons des parements seront done (écquations (60) :

$$
\operatorname{tg} \beta_{1}=0,175 \quad \operatorname{tg} \beta=0,745 \quad e_{0}=4 \mathrm{~m} .
$$

Les dimensions du profil seront alors :

$$
m=12^{\mathrm{m} 25} \quad m^{\prime}=52^{\mathrm{m} 15} \quad e=64^{\mathrm{m}} 40 \quad S=2305,0148
$$

Le fait d'adjoindre au parement aval la surface MNP diminue bien l'inclinaison de ce parement à la base. mais elle augmente aussi cette même inclinaison immédiatement en dessous du point $M$; il importe donc que ce point $M$ se trouve assez haut placé pour que l'augmentation du terme $\left(1+\operatorname{tg}^{2} \beta\right)$ ne soit pas gênante entre $M$ et $N$. Nous placerons en conséquence le point $\mathrm{M}$ à $30 \mathrm{~m}$. au-dessus de la base, de sorte que, avec une surface en segment de cercle, le point $\mathrm{N}$, ou l'inclination du parement redevient égale à 0,745 , ne se trouve à $55 \mathrm{~m}$. de profondeur.

En vérifiant les conditions de stabilité à la base on trouve :

$$
\begin{array}{lll}
\text { en charge : } & n^{\prime}=714299 & n^{\prime \prime}=1061703 \quad A=1331667 \\
\text { et à vide : } & n_{0}^{\prime}=851924 & A_{0}=1391714
\end{array}
$$

La prossion $n$ est supérieure à celle de l'eau, et la pros. sion maxima, aussi bien à vide qu'en charge, ne dépasse pas $14 \operatorname{ligs}$ par cme. Ces condition sont donc excellentes; toutefois on pourrait résuire légèrement $n$ 'et $A_{0}$.

Pour cela, on pourra diminuer un peu la valeur de $e$ (que nous avions forcéej en prenant $e=0,91 y$ et faire $\mu==0,193$. On aura alors $\operatorname{tg} \beta_{1}=0,175$ et $\operatorname{tg} \beta=0,735$.

Les dimensions plincipales seront alors :

$$
m=12 \mathrm{~m} 25 \quad m^{\prime}=51^{\mathrm{m} 45} \quad e=63^{\mathrm{m} 70} \quad S=2280,8719 \mathrm{~m}^{2}
$$

Ce qui donnera à vide : $\quad n_{0}^{\prime}=851936 \quad \Lambda_{0}=1391320$ et en charge: $\quad n^{\prime}=691380 \quad n^{\prime \prime}=10879_{1} \quad A=135402$

La valeur de $\Lambda_{0}$ est meilleure que précédemment, mais $n$ 'est trop faillle: nous avons trop diminué le paroment aval. En comparant les résultats obtenus dans les deux cas, on voit qu'il faudrait conserver $\operatorname{tg} \beta_{1}=0,175$ et prendre $\operatorname{tg} \beta=0,740$.

Supposons maintenant que l'on veuille adopter le profil $\dot{a}$ parement amont discontinu de la figure 75 . Nous admottrons que, jusqu'à $40 \mathrm{~m}$. de profondeur, le profil est établi avec parement amont réduit au minimum d'inclinaison. Nous admettrons en outre que le point $\mathrm{M}$ est encore à $30 \mathrm{~m}$. au-dessus de la base, c'est-à-dire qu'il se confond ici avec le point $\mathrm{E}$.

Nous avons icj: $H=70^{\mathrm{m}}, h=40^{\mathrm{m}}$ et $h "=30^{\mathrm{m}}$. Comme nous avions trouvé $c=0,90 y$, pour première approximation du profil précédent à parements rectilignes, nous ferons ici $m "=63^{m}$, de sorte que l'équation (70) devient :

$$
\frac{2,8}{1000} m^{3}-\left(m^{2}-32 m+185\right)=0
$$

Nous pouvons négliger sans grande errour le terme on $m^{3}$, car son coefficient est très petit par rapport à ceux dos autros termes; dès lors, de l'équation du second degré de la parenthèse on tire $m=7^{\mathrm{m}} 60$. Il est à remarcquer que la parenthèse prend une valeur négative lor'sque l'on donue it $m$ une valour légèremont plus grande; la racine de l'équation $3^{\text {mo }}$ degré est donc légèrement supúrieure à la valeur trouvée. Nous ferons ici $m=7 m 59$, ce qui correspond à une inclinaison de 0 m917 par mètre.

Nous déterminerons tgß de telle sorte que la surface du profil soit comprise entre 2280 et $2300 \mathrm{~m}^{2}$, ce qui nous conduit à adopter sur le joint EE' le rapport $e / y=0,88$ ( $n$ y est un peu plus grand que 1/9). Nous obtenons ainsi tg $\beta=0,853$ et $\operatorname{tg} \beta_{1}=0,027$ sur $40 \mathrm{~m}$. de hauteur, puis, sur les 30 derniers mètres, $\operatorname{tg} \beta_{1}^{\prime}=0,217$ à l'amont, avec inclinaison variable à l'aval suivant l'arc de cercle MNB.

Les dimensions principales seront alors : $e_{0}=4 \mathrm{~m}$

$$
m=7^{\mathrm{m} 59} \quad m^{\prime}=59,71 \quad e=67,30 \quad S=2296,2920
$$

Comme vérification des conditions de stabilıté, on trouve:

à vide : $\quad n_{0}^{\prime}=134060 \quad A_{0}=1101372$

en charge : $n^{\prime}=731712 \quad n^{\prime \prime}=9.4523 \quad \Lambda=1271226$.

La pression $n^{\prime}$ est plus forte que celle de l'eau, mais $A_{0}$ est trop fort. Nous pourrions, sans diminuer la surface du profil, diminuel légèrement la valeur do tg $\beta_{\beta}$ et augmenter cello de tg ${ }_{1}$, mais nous pouvons aussi modifier légèrement les résultats précédents sans modifier la section. En effet, si l'on incline un peu plus la corde MB du segment MNB, on déplacera vers l'aval le centre de gravité du triangle SPB, ce qui diminuera $n$ ' et $A_{0}$, peu toutefois, car la pression moyenne sera augmentée. Nous emploierons les deux pro- 
cédés à la fois. et nous ferons $\operatorname{tg} \beta=0,850, \operatorname{tg} \beta_{1}=0,026$, $\operatorname{tg} \beta_{1}=0,222$, en donnant à $\mathrm{MB}^{\prime}$ une inclinaison de $0 \mathrm{~m} 830$. Les dimensions principales seront alors : $e_{0}=1 \mathrm{~m}$.
$m=7 m 70$
$m \imath^{\prime}=58,90$
$e=66 \mathrm{~m} 60$
$S=2279,2075$

et comme vérification des conditions de stabilité on trouve: à vide : $\quad n_{0}^{\prime}=139237 \quad A_{0}=1391980$ en charge: $n^{\prime}=71901 \quad n^{\prime \prime}=97413$ $\Lambda=1281730$.

La compression maxima $A_{0}$ à vide est un peu inférieure à la limite fixée, toutcfois, on pourrait la réduire encore, en en même temps que $n$ et $S$, en diminuant légèrement $\operatorname{tg} \beta$ et en augmentant encore un peu l'inclinaison de AE ainsi que celle de la corte MB".

Si l'on compare les résultals obtenus avec les deux types do profil, on voit qu'ils sont à peu près ćcuivalents, toutefois, co dernier pourrait conduire à une très légère écono. mie dans le cube des maconneries, on faisant varier la largeur du couronnemont, ce qui pormettrait de réaliser les profils à section minima sur les flanes de la vallée où la hauteur serait inférieure à $40 \mathrm{~m}$. Mais, par contre, comme la largeur de la base est plus grande que dans le premier type, cette légère économie sur les maçonneries sera rapidement compenséc par le surcrolt de dépenses des fouilles dès que celles-ci devront être descendues un peu profond dans le sol.

Dans un prochain article nous terminerons en donnant quelques détails pratiques sup la construcíion des barrages.

H. Bellet.

\section{LE MOIS HYDRO-ÉLECTRIQUE}

\section{ACADÉMIE DES SCIENCES}

\section{GE்OLOGIE ET HYDROLOGIE}

Sur la " tenêtre ) du Plan-de-Nette et sur la géologie de la de la Haute Tarentaise. - Note de M. W. KillaN, Séance du 1er octobre 1906.

$\Lambda$ pon de distance an snd da col de la Leysse $\left(2650^{\mathrm{m}}\right)$, en Faute-Tarentarse, apparisissent des marbres roses of blancs, finement cristallns, avea interadahoms de banes lie de vin, également tros cristallus, dans lesquels la présence d'Apychus, de Bétemnites of the Phyllarmus, rencuntrés apres de longues recherches, nous a peruns de recomaitre le Juressigue superrenr it faches brinnconnass analogue au calcare de Guillestre, mas notablement plus cristallin. Ces curienses assises sonl arconnpagnces, comme dans coltams ponts du Briancommais, de breches culcares it coment rouge el blocs de matbre numr, rose el blane; elles se presentent dans los combhons suivantes : sur le versint sud du col de lat Leysse ot au pred du glaciel do lit Grande-Motto, on vol apparaltre sous les marbres phylliteux (T'las), bjen caractérisés, la succossion sulvante (de laaut on bas) :

8. Calcaures tratsiques gris

7. Calculures nankun it pite fine (Infralias ?) ;

6. Calearres norrs sehisteux et brèche calcaire breche du Tébsraphe), Isas ;

5. Marbres finement clistalins, blancs, roses, jaundres, avec bancs he de vm, arec Aphychus et traces d'aulres lossiles :

4. Bredhe in gros abments (hbes de marlores blancs, roses, ver-

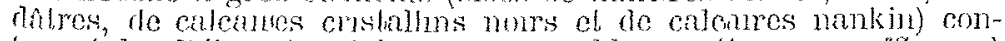
tenand, des bylemniles lres jecommassables : colte assise affeure it l'exh"imulé nord de la plano du Plan-de-Nelte, au point où le sentier franchit un petil escalpenent rochens:

3. Briehe calcaire à fragunculs do culcares gris, nombalres et jan-

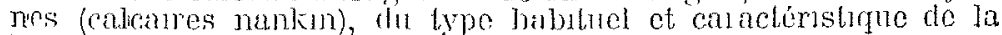
breche du Tógégraphe (Tals) du Prsauronnais;

2. Caleaire nankun it palo fino (lufridias ?);

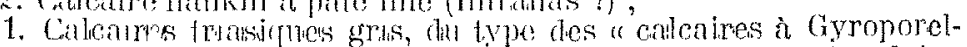
Ies ", visibles dans la barre rochenso qui limite au sud la plaine clu Planrde-Nette.

celte serie qui, de toute eridence, qeprésente une nappe spmall nale (1) (pli couchó) de Jurassique, a facies briançonnais, va plonger à l'est sous des Cargneules triasiques surmontées de schistes Justrés avec pirtre verdi (chaine do la Sana-Génépy). La racme de celte nappe (ou synchanal conché), qui plonge à l'ouesl sous le massil de la Grande-Mothe, doit otro cherchéce it l'ouest, c'est-i-dre dans la zone où règnenl lo factes brécholde du Lias et le type des marbres de Guillestre, contrairement a la masse de schistes lustrés qui la rcconvre et qui présente le facies piémontals, c'est-à-dire le type d'uno zone plus orientale.

Plus all nord, près de Tignes of de Val-d'Tsère, j'ai pu reconnaître igalement, dans les plis qu forment le soubassement din inassif de schistes lustrés de la Granrle-Sisssiòre, des noyaux synclinaux de miche (linsique) du Tólégraphe ot de marbres identıques à cenx dir Plan-de-Nelle.

Lit encore, par conséfucnt, on rencontre sous une masse, probablement charrice el reployfe de schistes hustros, tes plis couche's vers l'Jalie, à factes brianconnais. probablement autochtones. Ces plis, appartenant al flane est, de la zone axiale houillere, presentent les mênes facios al lu mène composfton gue ceux des onvirons de Mamrin, Grullostre, Nivache et ruo les synclinaux (GrantAréa) qu aceidontent l'évental houller ontre lia Gusane et l'Are Ils se continuent en Italie cutre le col de la Galise et la GrandeSassiore.

Il est réservé aux recherches futures de faire la part qui revient aux dépóts julassiques dans celle zone dans laquelle M. Marcel Bertrand, à qui nous devons d'avonr éclairé d'me vive lumiere la struclure de cetto haute région, n'avall signa é que des calcarres triasıques.

Sur le défaut d'étanchéité des zônes imperméables dans les sous-sols calcaires. - Note de M. F.-M. MARTEL, síance du $1^{\text {er }}$ octobre 1906.

En prósente des diffienllés lyrujéniques sonvent insurmontables auxquelles se heurtent actuelienent les projets de captage d'eau potable dans les régions calcares, on s'état pros à espérer que, clans cerhans cas, les rosurgences ponvalent etre naturellement protegtes, contie les infilialions polluantes, par des intercalations de zoncs marnenses aut-dessus an gisement géologique des émergences ; on aval pensé que ce tout mperméble, analogue à celui des caux artísicnnes, pourrat éxiter, an moms par places, les contammations proxmes. Dans une préfecture de l'ouest de la France, nne savante ítude géologique avait élabli que l'eau captée poup lalunenlation jailht des calcaires gréscux du Lias moyen, sous nue concho imperméable de marnes bleues (Lias superrieur) ćpaisse de $8^{\mathrm{m}}$ a. $10^{\mathrm{m}}$, couche supposce assez conlinue, assez etanche pour arrèter les diverses infiltratıons malsames recneillies par les calcaires fissutios (bajociens of bathoniens) suturs aurdessus. A la fin de 1905 , il n'en est pas noins smrvenu dans ladite ville une petite spretemie de fievre lyphodde, dont on n'a pu, parail-il, préciser l'élnologie.

Il me semble indispensabs, in ce propos, d'mdiquer que cetle hypothèse de lintanchélé des marnes intercalaires est contredile par la materiahlé des fauls, an moms pour les masses calcarres dont le niveau dépasse celui des plans de dramage exterienrs. Des 1889, dalleurs, avec G. Catupillat (Comples remdus, 25 novembre 1889), f'énoncais (pour les Causses) "qn'a travers la rone marneuse, lo conlenu des innombrables pelils róservorrs se déverse en sumtant par les gercures nalurelles "; lo dovelopiment des investugations soulerraines a de plus en plus confirmé que, meme dans les regions les moms disloqués, la contmuilé prolectrice des marnes no samral otro affirme nulle part. Ta plupart du temps, an contraire, los cassures des calcalres, inférleurs ou supérieurs anx zones marneuses, ont miéressét colles-ci au point de les interrompre sur toute leur épaisscur.

De plus, st argilense que soit une mame, il est absolument illusore de penser que sa dululion par les eaur souterraines parvienne it colmater les crevasses el à corriger les effels fachenx de la fissuration. Fu eflet (sans parler des grandes diaclases ou failles largement béantes it travers plusicurs assises) il est permas d'afflemer qu'en rigle générale, daus les calcaires, les eaux souterrumes (au-flessus loul au moins des plans de drainage exlérieurs qui provoquent lenrs emergences) ne sont pas slahques, mais dynamiques; après les pluies sultoul. (qui précisénent aminent les infiltrations dangereuses), leurs coumants plus on moins rapides et leurs colnnnes d'ean, ou conduntes forénes, sons forte pression hydrostatiqur, pénèrent ou pèsent dans les gercures do tonte espèce, an pont de fore unalérellement obstacle an tranquille el lent dópot de rósidi argitenx tuu seul pourrait plodnure le colmatage. Dans les grands avens des Causses. des Alpes, etc. (profonds de $100^{\mathrm{m}}$ à $200^{\mathrm{m}}$ ), le creusement des vallées sontirantes, en abaissant le niveau de base général et corrélativement cclui des eaux son-

(1) Le no 7 correspond au $n^{\circ} 2$; lo n० 6 au $n^{\circ} 3 ; 10 n^{\circ} 4$ et le no 5 repró sentent lo noyau du synclinal. Cet ensemble plonge de toutes parts fous dos assises plus anciennes et apparalt comme par une a fenetre n dans la dépresaion du Plan-de-Netta gur les bonds de laquelle, notam. ment lextémite nond, on peut alsément l'étudian. 